\title{
A BOUNDED HILBERTIAN BASIS IN $C[0,1]$
}

\author{
SHERWOOD SAMN
}

Abstract. The existence of a bounded Hilbertian basis in $C[0,1]$ is established.

1. Introduction. It is well known that an orthonormal sequence $\left\{x_{n}\right\}$ in a Hilbert space possesses the following properties:

(1) $\sum_{i=1}^{\infty} a_{i} x_{i}$ converges implies $\sum_{i=1}^{\infty}\left|a_{i}\right|^{2}<\infty$, and

(2) $\sum_{i=1}^{\infty}\left|a_{i}\right|^{2}$ implies $\sum_{i=1}^{\infty} a_{i} x_{i}$ converges.

A basis $\left\{x_{i}\right\}$ in a Banach space satisfying (1) is called a Besselian basis and a basis $\left\{x_{i}\right\}$ in a Banach space satisfying (2) is called an Hilbertian basis. A basis $\left\{x_{n}\right\}$ is bounded if $0<\inf _{n}\left\|x_{n}\right\| \leqq \sup _{n}\left\|x_{n}\right\|<\infty$. In [2], Pełczyński raised the question whether there exists in $C[0,1]$ (in $L^{1}[0,1]$ ) a bounded Besselian (resp. Hilbertian) basis, in particular, whether there exists in $C[0,1]$ a bounded orthonormal basis. In [4], Olevskil proved the nonexistence of bounded orthonormal basis in $C[0,1]$; in this paper we will show that there exists a bounded Hilbertian basis in $C[0,1]$.

A basis $\left\{x_{n}\right\}$ of a Banach space is $w c_{0}$ (or semishrinking) if $x_{n}$ converges weakly to 0 . Since every Hilbertian basis is a $w c_{0}$ basis, it is natural to look for a bounded Hilbertian basis in the class of bounded $w c_{0}$ bases. The referee has pointed out to us that Warren [3] has constructed a bounded $w c_{0}$ basis in $C[0,1]$. Our basis is similar to his, but is a little simpler.

2. Main result. Let $a_{0}=0, a_{1}=1$ and for $j=2^{n}, a_{j+k}=(2 k-1) /(2 j)$ $\left(n=0,1, \cdots ; k=1,2, \cdots, 2^{n}\right)$. Let $b_{n}=1 / 2^{n+1}(n=1,2, \cdots)$ and let $\left\{c_{n}\right\}$ be the subsequence of $\left\{a_{n}\right\}$ complementary to the subsequence $a_{0}, a_{1}, b_{1}, b_{2}, \cdots$ of $\left\{a_{n}\right\}, \quad$ i.e. $\quad c_{1}=\frac{1}{2}, \quad c_{2}=\frac{3}{4}, \quad c_{3}=\frac{3}{8}, \quad c_{4}=\frac{5}{8}, \quad c_{5}=\frac{7}{8}$, $c_{6}=\frac{3}{16}, \cdots$. Next we define a rearrangement of $\left\{a_{n}\right\}$ as follows: $a_{0}, a_{1}, c_{1}$; $b_{1}, b_{2}, b_{3}, c_{2} ; \cdots ; b_{q(n-1)+1}, \cdots, b_{q(n)}, c_{n} ; \cdots$, where $q(n)=1+2^{2}+\cdots+$ $n^{2}-n(n=2,3, \cdots)$. (Note. To each positive integer $n$ is associated a group of numbers consisting of $n^{2}-1 b_{i}$ 's and one $c_{i}$.) To simplify matters, let us rename the above sequence as $d_{0}, d_{1}, d_{2}, \cdots$.

We now define a sequence in $C[0,1]$ as follows (it is slightly different

Received by the editors June 22, 1972 and, in revised form, September 13, 1972 and January 17, 1973.

AMS (MOS) subject classifications (1970). Primary 46B15; Secondary 46E15.

(c) American Mathematical Society 1973 
from the usual way of defining a generalized Schauder basis $[1$, p. 11]):

and for $n \geqq 3$

$$
\begin{array}{rlrl}
x_{0}(t) & =1, & & \\
x_{1}(t) & =1-t, & \\
x_{2}(t) & =1, & & t=d_{2}, \\
& =0, & & t \in\left\{d_{0}, d_{1}\right\}, \\
& =\text { linear } & & \text { for other } t,
\end{array}
$$

$$
\begin{aligned}
x_{n}(t) & =1, & & t=d_{n}, \\
& =0, & & t \in\left\{d_{0}, d_{1}, \cdots, d_{n-1}, g_{n}\right\}, \\
& =\text { linear } & & \text { for other } t,
\end{aligned}
$$

where $g_{n}$ denotes the first $b_{i}$ in the sequence $\left\{b_{i}\right\}$ not in $\left\{d_{0}, d_{1}, \cdots, d_{n}\right\}$.

Finally, we define another sequence in $C[0,1]$ as follows: $y_{i}=x_{i}$ $(i=0,1,2)$, and for $n \geqq 2$ and $p(n)=1+2^{2}+\cdots+(n-1)^{2}+1$

$$
\begin{aligned}
& y_{p(n)+1}=-x_{p(n)+1}-x_{p(n)+2}-\cdots-x_{p(n+1)-1}+n^{-2} x_{p(n+1)}, \\
& y_{p(n)+k}=x_{p(n)+k-1}+n^{-2} x_{p(n+1)} \quad\left(k=2, \cdots, n^{2}\right) .
\end{aligned}
$$

We note in particular that for $n \geqq 3,1 \leqq k \leqq n^{2}$, the support of $y_{p(n)+k}$ is contained in the union of two closed disjoint intervals $I_{n, 1}$ and $I_{n, 2}$, where $I_{n, 1}=\left[b_{q(n)+1}, b_{q(n-1)}\right]$ and $I_{n, 2}=\left[b_{q(n-1)-1}, 1\right]$. (Note. $b_{q(n-1)-1} \leqq$ $b_{n-1}<c_{n}<1$.)

THEOREM. The sequence $\left\{y_{n}\right\}$ is a bounded Hilbertian basis of $C[0,1]$.

Proof. The direct method used in showing the generalized Scauder basis of $C[0,1]$ is a basis $[1, \mathrm{p} .11]$ can be used with slight modification to show $\left\{x_{n}\right\}$ is a basis. Since $x_{i}=y_{i}(i=0,1,2), x_{p(n+1)}=y_{p(n)+1}+\cdots+$ $y_{p(n+1)}, \quad$ and $x_{p, n)+k}=y_{p(n)+k+1}-n^{-2}\left(y_{p(n)+1}+\cdots+y_{p(n+1)}\right) \quad(k=1, \cdots$, $\left.n^{2}-1\right),\left[x_{i}\right]=\left[y_{i}\right](i=0,1,2)$ and $\left[x_{p(n)+1}, \cdots, x_{p(n+1)}\right]=\left[y_{p(n)+1}, \cdots\right.$, $\left.y_{p(n+1)}\right]$. Hence it suffices $[1$, p. 64] to show that there is a constant $C$ independent of $n$ such that for any sequence of real numbers $h_{1}, h_{2}, \cdots$,

$$
\left\|\sum_{i=1}^{k} h_{i} y_{p(n)+i}\right\| \leqq C\left\|\sum_{i=1}^{n^{2}} h_{i} y_{p(n)+i}\right\|
$$

$\left(n=1,2, \cdots ; k=1,2, \cdots, n^{2}\right)$.

The following argument is similar to an argument used in [3]; we present it here for completeness. Let $g(k)=0$ if $k=n^{2}$ and $=1$ if $1 \leqq k<n^{2}$, then

$$
\begin{aligned}
\left\|\sum_{i=1}^{k} h_{i} y_{p(n)+i}\right\| & \\
& =\operatorname{Max}\left\{\left|h_{1}+\cdots+h_{k}\right| / n^{2},\left|h_{1}-h_{2}\right|, \cdots,\left|h_{1}-h_{k}\right|, g(k)\left|h_{1}\right|\right\} \\
& \leqq 2 \operatorname{Max}\left\{\left|h_{i}\right| \mid 1 \leqq i \leqq n^{2}\right\} .
\end{aligned}
$$


Now let $e_{1}=\left(h_{1}+\cdots+h_{N}\right) / N$, and $e_{k}=h_{k}-h_{1}, k=2, \cdots, n^{2}=N$; then $h_{1}=e_{1}-\left(e_{1}+e_{2}+\cdots+e_{n}\right) / N, h_{k}=e_{k}+h_{1}\left(k=2, \cdots, n^{2}=N\right)$, and

$$
\operatorname{Max}\left\{\left|h_{i}\right| \mid 1 \leqq i \leqq n^{2}\right\} \leqq 3 \operatorname{Max}\left\{\left|e_{i}\right| \mid 1 \leqq i \leqq n^{2}\right\} .
$$

Hence for $N=n^{2}$

$$
\begin{aligned}
\left\|\sum_{i=1}^{N} h_{i} y_{p(n)+i}\right\| & =\operatorname{Max}\left\{\left|h_{1}+\cdots+h_{N}\right| / N,\left|h_{1}-h_{2}\right|, \cdots,\left|h_{1}-h_{N}\right|\right\} \\
& =\operatorname{Max}\left\{\left|e_{i}\right| \mid 1 \leqq i \leqq N\right\} \\
& \geqq \frac{1}{3} \operatorname{Max}\left\{\left|h_{i}\right| \mid 1 \leqq i \leqq N\right\} \\
& \geqq \frac{1}{6}\left\|\sum_{i=1}^{k} h_{i} y_{p(n)+i}\right\| .
\end{aligned}
$$

Hence $C$ can be chosen to be 6 .

To show that $\left\{y_{n}\right\}$ is a bounded Hilbertian basis, we may disregard a finite number of $y_{i}$ 's. Therefore, for sake of symmetry, we will consider only $y_{p(n)+k}$ for $n \geqq 3,1 \leqq k \leqq n^{2}$. Let $I_{n, 1}$ and $I_{n, 2}$ be the two disjoint closed intervals mentioned above. It is clear that

$$
\sup \left\{\left|y_{p(n)+k}(t)\right| \mid t \in I_{n, 1}\right\}=1,
$$

and

$$
\sup \left\{\left|y_{p(n)+k}(t)\right| \mid t \in I_{n, 2}\right\}=n^{-2} \quad\left(n \geqq 3,1 \leqq k \leqq n^{2}\right) .
$$

For $n \geqq 3,1 \leqq k \leqq n^{2}$, let

$$
\begin{aligned}
u_{p(n)+k}(t) & =y_{p(n)+\cdot k}(t), & & t \in I_{n .1}, \\
& =0 & & \text { otherwise, } \\
v_{p(n)+k}(t) & =y_{p(n)+k}(t), & & t \in I_{n .2}, \\
& =0 & & \text { otherwise. }
\end{aligned}
$$

One can readily verify that $u_{p(n)+1}=-\left(x_{p(n)+1}+\cdots+x_{p(n+1)-1}\right), u_{p(n)+k}=$ $x_{p(n)+k-1}\left(k=2, \cdots, n^{2}\right)$, and $v_{p(n)+k}=n^{-2} x_{p(n+1)}\left(k=1,2, \cdots, n^{2}\right)$. Thus $u_{p(n)+k}, v_{p(n)+k}$ are in $C[0,1], u_{p(n)+k}+v_{p(n)+k}=y_{p(n)+k},\left\|u_{p(n)+k}\right\|=1$, $\left\|v_{p(n)+k}\right\|=n^{-2}, u_{p(n)+1} \leqq 0$ and $u_{p(n)+k} \geqq 0\left(2 \leqq k \leqq n^{2}\right)$. And because of the way the $x_{i}$ 's are defined, we also have

$$
\left\|\sum_{n=3}^{N} u_{p(n)+1}\right\|=\left\|\sum_{n=3}^{N} \sum_{j=1}^{n^{2}-1} x_{p(n)+j}\right\|=1
$$


$(N \geqq 3)$, and similarly,

$$
\left\|\sum_{n=3}^{N-1} \sum_{k=2}^{n^{2}} u_{p(n)+k}+\sum_{k=2}^{K} u_{p(N)+k}\right\|=1
$$

$\left(N \geqq 3,2 \leqq K \leqq N^{2}\right)$.

Let $f \in C[0,1]^{*}$. Then $f\left(y_{n}\right)=f\left(u_{n}\right)+f\left(v_{n}\right)(n \geqq 7)$, and

$$
\sum_{n=7}^{\infty}\left|f\left(y_{n}\right)\right|^{2} \leqq 2\left\{\sum_{n=7}^{\infty}\left|f\left(u_{n}\right)\right|^{2}+\sum_{n=7}^{\infty}\left|f\left(v_{n}\right)\right|^{2}\right\} .
$$

Hence it is sufficient to prove the two series on the right converge. Now $\left|f\left(v_{n}\right)\right| \leqq\|f\|\left\|v_{n}\right\|=\|f\| / k^{2}$ for some $k \geqq 3$. But because of the way the $y_{n}$ 's are constructed, there are exactly $k^{2} v_{n}$ 's with $\left\|v_{n}\right\|=k^{-2}$. Hence

$$
\sum_{n=7}^{\infty}\left|f\left(v_{n}\right)\right|^{2} \leqq\|f\|^{2} \sum_{k=3}^{\infty} k^{2}\left(\frac{1}{k^{2}}\right)^{2}<\infty .
$$

To show the other series converges, we note that there exists some function $h$ of bounded variation on $[0,1]$ such that $f\left(u_{n}\right)=\int_{0}^{1} u_{n} d h$. Let $h_{1}$ and $h_{2}$ be the positive and negative variations of $h$ respectively. For any integer $M=p(N)+K \geqq 7\left(N \geqq 3,1 \leqq K \leqq N^{2}\right)$,

$$
\begin{aligned}
\sum_{n=7}^{M}\left|f\left(u_{n}\right)\right|= & \sum_{n=3}^{N-1} \sum_{k=1}^{n^{2}}\left|f\left(u_{p(n)+k}\right)\right|+\sum_{k=1}^{K}\left|f\left(u_{p(N)+k}\right)\right| \\
= & \sum_{n=3}^{N}\left|f\left(u_{p(n)+1}\right)\right|+\sum_{n=3}^{N-1} \sum_{k=2}^{n}\left|f\left(u_{p(n)+k}\right)\right|+\sum_{k=2}^{K}\left|f\left(u_{p(N)+k}\right)\right| \\
\leqq & \sum_{i=1}^{-2} \sum_{n=3}^{N}\left|\int_{0}^{1} u_{p(n)+1} d h_{i}\right| \\
& +\sum_{i=1}^{2}\left\{\sum_{n=3}^{N-1} \sum_{k=2}^{n^{2}}\left|\int_{0}^{1} u_{p(n)+k} d h_{i}\right|+\sum_{k=2}^{K}\left|\int_{0}^{1} u_{p(N)+k} d h_{i}\right|\right\} \\
= & \sum_{i=1}^{2}\left|\int_{0}^{1}\left(\sum_{n=3}^{N} u_{p(n)+1}\right) d h_{i}\right| \\
& +\sum_{i=1}^{2}\left|\int_{0}^{1}\left(\sum_{n=3}^{N-1} \sum_{k=2}^{n^{2}} u_{p(n)+k}+\sum_{k=2}^{K} u_{p(N)+k}\right) d h_{i}\right| \\
\leqq & 2\left(V\left(h_{1}\right)+V\left(h_{2}\right)\right)
\end{aligned}
$$

where $V\left(h_{i}\right)(i=1,2)$ are the total variations of the monotone increasing functions $h_{i}$. This obviously implies the convergence of the series.

Finally, the boundedness is clear. This completes the proof. 


\section{REFERENCES}

1. I. Singer, Bases in Banach spaces. I, Springer, Berlin and New York, 1970.

2. A. Pelczyński, Some problems on bases in Banach and Fréchet spaces, Israel J. Math. 2 (1964), 132-138. MR 30 \#3356.

3. H. Warren, A special basis for $C[0,1]$, Proc. Amer. Math. Soc. 27 (1971), 495-499. MR 42 \#5023.

4. A. M. Olevskiĭ, Fourier series of continuous functions with respect to bounded orthonormal systems, Izv. Akad. Nauk SSSR Ser. Mat. 30 (1966), 387-432. (Russian) MR 33 \#4581.

Department of Mathematical Sciences, Indiana and Purdue Universities at INDIANAPOLIS, INDIANAPOLIS, INDIANA 46205 